04.1

\title{
Нелинейное взаимодействие электромагнитных волн в плотной плазме
}

\author{
(C) Г.А. Павлов \\ Институт проблем химической фризики РАН, Черноголовка, Московская обл., Россия \\ E-mail: pav14411@yandex.ru
}

Поступило в Редакцию 6 декабря 2019 г.

В окончательной редакции 22 января 2020 г.

Принято к публикации 31 января 2020 г.

\begin{abstract}
Рассмотрены нелинейные явления, вызванные квадратичным взаимодействием электромагнитных волн в плотной заряженной среде (кулоновские системы, плазма): параметрическая генерация и генерация второй гармоники электромагнитного излучения. Для определения квадратичных функций реакции, описывающих взаимодействия электромагнитных волн в среде, применен подход, основанный на использовании явных аппроксимаций для функций реакции с подгоночными параметрами. Параметры найдены из точных частотных моментов функций реакции. С использованием данных по функциям реакции выполнена оценка условий экспериментальной реализации перечисленных явлений в лабораторной плотной плазме в постоянном магнитном поле.
\end{abstract}

Ключевые слова: нелинейные явления, плотная плазма.

DOI: 10.21883/PJTF.2020.08.49311.18143

В последние несколько лет проводятся интенсивные исследования генерации второй гармоники и других нелинейных явлений, обусловленных квадратичным взаимодействием электромагнитных волн в различных средах: кристаллах, графене, различных смесях, композитах, слабоионизованных газах, суспензиях и т.д. (см., например, [1-4]). В то же время аналогичные явления в плотной плазме и других плотных заряженных средах, нелинейные свойства этих сред теоретически изучены фрагментарно. Рассмотрены лишь нелинейные переносные и гидродинамические характеристики таких сред, а также их нелинейная реакция на продольное электрическое поле: плазменное эхо и преобразование волн $[5,6]$. Экспериментальные работы по нелинейным явлениям в плотной плазме и ее свойствам отсутствуют. Эти очевидные пробелы в исследованиях нелинейных характеристик плотных заряженных сред определяют актуальность настоящей работы, посвященной изучению квадратичного взаимодействия электромагнитных волн в плотной плазме. Заметим, что линейные характеристики таких сред подробно изучались экспериментально и теоретически в течение предшествующих 50 лет (см., например, [7,8]).

Рассмотрим параметрическую генерацию и генерацию второй гармоники электромагнитного излучения (ПГИ и ГВГ соответственно), которые представляют собой результаты квадратичного взаимодействия электромагнитных волн при распространении в плотной (неидеальной) плазме в постоянном магнитном поле $\mathbf{B}_{0}$. ПГИ характеризуется перекачкой энергии к двум относительно слабым электромагнитным волнам - „сигнальной“ $(\mathrm{CB})$ и „холостой“ $(\mathrm{XB})$ с частотами $\omega_{1}$ и $\omega_{2}$ - от интенсивной электромагнитной „волны накачки“ $(\mathrm{BH})$ с частотой $\omega_{3}$. ГВГ есть эффект квадратичного взаимодействия двух волн на частоте $\omega$ в среде (или переизлучение на частоте $2 \omega$ поляризованной средой, вызванное одной волной с частотой $\omega$ ), результатом которого является генерация электромагнитной волны на частоте $2 \omega$. Исследуем условия реализации ПГИ (исследование ГВГ во многом аналогично), учитывая особенности тензоров квадратичной восприимчивости $\chi_{i j k}^{(2)}$ и диэлектрической проницаемости $\varepsilon_{i j}$ в плотной плазме и поле $\mathbf{B}_{0}$, оценим возможность соответствующих экспериментальных исследований. Полагаем, что $\chi_{i j k}^{(2)}$ и $\varepsilon_{i j}$ не зависят от интенсивности ВН. Как и в [6], далее для определения $\chi_{i j k}^{(2)}$, комплексной функции, применен подход, основанный на использовании явных аппроксимаций данной функции с подгоночными параметрами, которые находятся из точных частотных моментов $\chi_{i j k}^{(2)}$.

Для исследования ПГИ необходимы уравнения для амплитуд $A_{1}(z), A_{2}(z), A_{3}(z)$ трех взаимодействующих электромагнитных волн с частотами и волновыми векторами $\omega_{1}, \mathbf{k}_{1} ; \omega_{2}, \mathbf{k}_{2} ; \omega_{3}, \mathbf{k}_{3}$ [9]. Данные уравнения рассмотрим для однородной плазмы во внешнем постоянном и однородном магнитном поле с учетом нелинейной поляризации среды $\mathbf{P}_{n l}$, заданной соотношением $P_{n l, i}(\mathbf{r}, t)=\chi_{i j k}^{(2)} E_{j}(\mathbf{r}, t) E_{k}(\mathbf{r}, t)$. Линейная часть поляризации включена в диэлектрическую проницаемость $\varepsilon_{i j}$. Поле в среде $\mathbf{E}(z, t)$ является суперпозицией полей $\mathrm{CB}$, $\mathrm{XB} \quad$ и $\mathrm{BH}: \quad \mathbf{E}(z, t)=(1 / 2) \sum_{n=1}^{3}\left[\mathbf{e}_{n} A_{n}(z) e^{i\left(\omega_{n} t-\mathbf{k}_{n} \cdot \mathbf{z}\right)}+c . c.\right]$ $\left(n=1-3, \mathbf{E}_{n}(z, t)=\mathbf{e}_{n} A_{n}(z) e^{i\left(\omega_{n} t-\mathbf{k}_{n} \cdot \mathbf{z}\right)}, \mathbf{e}_{n}-\right.$ единичный вектор поляризации волны на частоте $\omega_{n},\left\{\mathbf{e}_{n, x y z}\right\}-$ набор компонентов вектора $\mathbf{e}_{n}$ ). Далее используем приближение медленно меняющихся во времени и про- 
странстве амплитуд волн. При $\chi^{(2)} \approx \operatorname{Re} \chi^{(2)}$ и заданном поле „накачки“ (когда $a_{1,2}(z) \ll a_{3}(0)$ волны накачки и амплитуда $a_{3}$ уменьшается только из-за поглощения $\delta_{3}$ в среде, а не из-за перекачки энергии в СВ и ХВ; см. обозначения (3)) положим, что $\delta_{n}=\delta, \omega_{1}=\omega_{2}$, $\omega_{3}=2 \omega_{1}, \sigma_{1}=\sigma_{2}, a_{1}=a_{2}, \Psi(z) \approx \pi / 2+\Delta \mathbf{k} \cdot \mathbf{z}$. После этих непринципиальных упрощений $a_{1}$ примет вид

$$
a_{1}(z)=a_{1}(0) \exp \left\{\left[\operatorname{Re} \sigma_{1} a_{3}(0) \frac{\sin (\Delta k z)}{\Delta k z}-\delta_{1}\right] z\right\} .
$$

В случае $\sigma_{n} \approx \operatorname{Im} \sigma_{n}$ (т.е. $\left.\chi^{(2)} \approx i \operatorname{Im} \chi^{(2)}\right)$ вместо (1) найдем

$$
a_{1}(z)=a_{1}(0) \exp \left\{\left[\operatorname{Im} \sigma_{1} a_{3}(0) \frac{\sin (\Delta k z)}{\Delta k z}-\delta_{1}\right] z\right\} .
$$

В выражениях (1) и (2), удобных для анализа условий реализации ПГИ в среде, введены следующие обозначения в связи с переходом от комплексных амплитуд $A_{1,2,3}(z)$ к вещественным амплитудам и фазам, $\Delta \mathbf{k}=\mathbf{k}_{3}-\mathbf{k}_{1}-\mathbf{k}_{2}, \Delta k$ - проекция $\Delta \mathbf{k}$ на ось $z, n(\omega)-$ показатель преломления на частоте $\omega$ :

$$
\begin{gathered}
A_{n}(z)=a_{n} e^{i \varphi_{n}(z)}, \quad \Psi \equiv \varphi_{3}-\varphi_{1}-\varphi_{2}-\Delta \mathbf{k} \cdot \mathbf{z}, \\
\delta_{n}=\omega_{n} \mathbf{e}_{n} \frac{\operatorname{Im} \varepsilon\left(\omega_{n}\right) \mathbf{e}_{n}}{2 n\left(\omega_{n}\right) c}, \quad k=\omega n(\omega) / c \\
\sigma_{1}=2 \pi \omega_{1} \mathbf{e}_{1} \frac{\chi^{(2)}\left(\omega_{1}\right): \mathbf{e}_{2} \mathbf{e}_{3}}{n\left(\omega_{1}\right) c} \\
\sigma_{2}=2 \pi \omega_{2} \mathbf{e}_{2} \frac{\chi^{(2)}\left(\omega_{2}\right): \mathbf{e}_{1} \mathbf{e}_{3}}{n\left(\omega_{2}\right) c} \\
\sigma_{3}=2 \pi \omega_{3} \mathbf{e}_{3} \frac{\chi^{(2)}\left(\omega_{3}\right): \mathbf{e}_{1} \mathbf{e}_{2}}{n\left(\omega_{3}\right) c} .
\end{gathered}
$$

$\mathrm{B}(3) \delta_{n}$ и $\sigma_{n}$ представляют собой свертки тензоров $\varepsilon_{i j}$ и $\chi_{i j k}^{(2)}$ с векторами поляризации по соответствующим индексам.

Таким образом, условиями экспоненциального роста амплитуды СВ, следующими из (1) и (2), являются положительность действительной или мнимой частей $\sigma_{1}$; $\operatorname{Re} \sigma_{1} a_{3}(0)>\delta_{1}, \operatorname{Im} \sigma_{1} a_{3}(0)>\delta_{1} \quad$ (и $\left.\Delta \mathbf{k} \cdot \mathbf{z}=0\right)$. Нарастание амплитуды $\mathrm{CB}$ по $z$ также возможно, когда вместо (1) (или (2)) используется более полная система уравнений для амплитуд и $\operatorname{Re} \sigma_{1} a_{3}(0)>\Delta k(f=2$, см. таблицу); то же для $\operatorname{Im} \sigma_{1} a_{3}(0)>\Delta k$. Выполнение и конкретизация перечисленных условий зависят от интенсивности и поляризации взаимодействующих электромагнитных волн (свертки в определениях (3) не должны быть равны нулю), вида и значений действительной и мнимой частей тензора $\chi_{i j k}^{(2)}, \mathbf{B}_{0}$ и линейных свойств среды $\left(\delta_{1}\right.$, см. таблицу). Заметим, что линейные динамические свойства $-\varepsilon_{i j}-$ плотной заряженной среды достаточно хорошо изучены (см., например, $[7,8]$ ), но нелинейная реакция такой среды на электромагнитное поле не исследовалась. В изотропной плазме параметры $\left\{\sigma_{n}\right\}$ равны нулю. В случае ГВГ одной волной основной частоты с амплитудой $a_{1}$ можно получить для амплитуды второй гармоники $a_{2}$ (по аналогии с (1), (2)) $a_{2}(z) \approx \sigma_{2} a_{1}^{2} z$ (на длине когерентности, если $\sigma_{i} \approx \operatorname{Re} \sigma_{i}$ и $\left.\sigma_{2} a_{1}^{2} \cos (\Delta k z)-\delta_{2} a_{2}>0\right)$.

Рассмотрим тензор квадратичной восприимчивости $\chi_{i j k}^{(2)}$ анизотропной плотной плазмы (в постоянном магнитном поле $\left.\mathbf{B}_{0}\right)$, свойства которого определяют условия реализации ПГИ в данной среде. Можно получить формальный вид $\chi_{i j k}^{(2)}$ через соответствующие корреляторы методами теории нелинейного отклика (см., например, [6]). Но, очевидно, что для плотной (неидеальной в отличие от разреженной плазмы) последовательное вычисление $\chi_{i j k}^{(2)}$ невозможно, так как отсутствует малый параметр по межчастичному взаимодействию $(\Gamma \geq 1$, $\left.\Gamma=e^{2} / a k_{B} T, a=(3 / 4 \pi n)^{1 / 3}\right)$. Поэтому в работе используем модельный подход, заключающийся в применении явной аппроксимации с подгоночными параметрами для $\chi_{i j k}^{(2)}$, параметры удобно найти из точных частотных моментов $\chi_{i j k}^{(2)}$ [6]. Выпишем аппроксимацию $\chi_{i j k}^{(2)}$ анизотропной плотной высокотемпературной заряженной среды (при $n \lambda^{3} \ll 1, \lambda-$ длина волны де Бройля) в форме, соответствующей решению уравнения Власова (см., например, [10]) с одним подгоночным параметром $(v-$ эффективная частота столкновений):

$$
\begin{aligned}
& \chi_{i j k}^{(2)}\left(\omega_{1}, \mathbf{k}_{1} ; \omega_{2}, \mathbf{k}_{2}\right)=\sum_{l} \frac{-i}{2} \frac{\omega_{p l}^{2} e_{l}}{m_{l}} \frac{1}{\omega_{1} \omega_{2} \omega} \int d \mathbf{v} \\
& \times \sum_{n} \frac{e^{i n \varphi-i a \sin \varphi}}{\omega-k_{\|} v_{\|}-n \omega_{B}+i v} X_{i}^{(n)}\left(\mathbf{k}_{\perp}, \mathbf{v}\right)\left[Y_{j}\left(\omega_{1}, \mathbf{k}_{1}, \mathbf{v}\right)\right. \\
& \times \sum_{n_{2}} \frac{e^{-i n_{2}\left(\varphi-\varphi_{2}\right)+i a_{2} \sin \left(\varphi-\varphi_{2}\right)}}{\omega_{2}-k_{2 \|} v_{\|}-n_{2} \omega_{B}+i v} Z_{k}^{\left(n_{2}\right)}\left(\omega_{2}, \mathbf{k}_{2}, \mathbf{v}\right) \\
& +Y_{k}\left(\omega_{2}, \mathbf{k}_{2}, \mathbf{v}\right) \sum_{n_{1}} \frac{e^{-i n_{1}\left(\varphi-\varphi_{1}\right)+i a_{1} \sin \left(\varphi-\varphi_{1}\right)}}{\omega_{1}-k_{1 \|} v_{\|}-n_{1} \omega_{B}+i \nu} \\
& \left.\times Z_{j}^{\left(n_{1}\right)}\left(\omega_{1}, \mathbf{k}_{1}, \mathbf{v}\right)\right] f_{0}\left(v_{\|}, v_{\perp}\right), \\
& X_{i}^{(n)}=\left|\begin{array}{c}
\left(n \omega_{B} / k_{\perp}\right) J_{n}(a) \cos \varphi_{k}+i v_{\perp} J_{n}^{\prime}(a) \sin \varphi_{k} \\
\left(n \omega_{B} / k_{\perp}\right) J_{n}(a) \sin \varphi_{k}-i v_{\perp} J_{n}^{\prime}(a) \cos \varphi_{k} \\
v_{\|} J_{n}(a)
\end{array}\right|, \\
& Y_{i}=\left[\left(\omega_{1}-\mathbf{k}_{1} \mathbf{v}\right) \delta_{i j}+k_{1 j} v_{i}\right] \frac{\partial}{\partial v_{j}}, \\
& Z_{i}^{(n)}=X_{i}^{(n)^{*}}\left(\frac{\omega-k_{\|} v_{\|}}{v_{\perp}} \frac{\partial}{\partial v_{\perp}}+k_{\|} \frac{\partial}{\partial v_{\|}}\right)-\delta_{i z} J_{n}(a) \\
& \times\left(\omega-k_{\|} v_{\|}-n \omega_{B}\right)\left(\frac{v_{\|}}{v_{\perp}} \frac{\partial}{\partial v_{\perp}}-\frac{\partial}{\partial v_{\|}}\right) .
\end{aligned}
$$

В (4) $f_{0}$ - функция распределения Максвелла; $e_{i}, m_{i}, \omega_{p i}$ - заряд, масса и плазменная частота $i$-го 
Параметры ПГИ для лабораторной плазмы (см. (1), (3); $f=a_{3}(0) \operatorname{Re} \sigma_{1} / \Delta k, \quad \omega_{1}=\omega_{2}=\omega_{3} / 2, \quad r_{S}=\left(a_{3}(0) \operatorname{Re} \sigma_{1}\right)^{-1}$, $\left.s=a_{3}(0) \operatorname{Re} \sigma_{1}-\delta_{1}\right)$

\begin{tabular}{c|c|c|c|c|c|c|c|c|c}
\hline$B_{0}, \mathrm{G}$ & $T, \mathrm{eV}$ & $n_{e}, 10^{21} \mathrm{~cm}^{-3}$ & $v / \omega_{p}$ & $a_{3}(0), \mathrm{V} / \mathrm{cm}$ & $\omega_{1} / \omega_{p}$ & $\delta_{1}, \mathrm{~cm}^{-1}$ & $\operatorname{Re} \sigma_{1}, \mathrm{~V}^{-1}$ & \multicolumn{2}{|c}{$s, \mathrm{~cm}^{-1} ; r_{S}, \mathrm{~cm}$} \\
\hline $10^{4}$ & 3 & 1 & $10^{-2}$ & $10^{6}-10^{7}$ & 1.6 & $2.4 \cdot 10^{2}$ & $10^{-10}$ & $f=0$ & $s<0$ \\
$10^{4}$ & 3 & $10^{-3}$ & $10^{-4}$ & $10^{6}-10^{7}$ & 1.6 & 0.25 & $2 \cdot 10^{-9}$ & $s<0$ \\
$10^{5}$ & 3 & $10^{-5}$ & $10^{-5}$ & $10^{6}-10^{7}$ & 1.6 & 0.003 & $2 \cdot 10^{-7}$ & $r_{S} \sim 5-0.5 \mid \tilde{r}_{S} \sim 6.5-0.6$
\end{tabular}

сорта частиц, суммирование проводится по всем сортам заряженных частиц среды; $\varphi, \varphi_{1}, \varphi_{2}$ - азимутальные углы векторов $\mathbf{v}, \mathbf{k}_{1}, \mathbf{k}_{2}$ в плоскости, перпендикулярной $\mathbf{B}_{0}$; система координат - ось $z \| \mathbf{B}_{0}$, ось $x$ лежит в плоскости $\mathbf{k}, \mathbf{B}_{0} ; J_{n}(a)-$ функция Бесселя $n$-го порядка, $a=k_{\perp} v_{\perp} / \omega_{B}, \omega_{B}=e B_{0} / m c$. Далее для (4) используем значения $v$ из [6], т.е. данный параметр найден для заданной комбинации $\left\{\mathbf{k}_{i}\right\}$ при отсутствии внешнего поля $\left(\mathbf{B}_{0}=0\right.$, см. таблицу).

Для оценки параметров $\left\{\sigma_{n}\right\}$ в (1), (2) зададим конфигурацию $\left\{\mathbf{k}_{i}\right\}$ и $\left\{\mathbf{e}_{i}\right\}$ следующим образом: $\mathbf{k} \equiv \mathbf{k}_{3} \| \mathbf{B}_{0}$, $\left\{\mathbf{k}_{i}\right\}$ находятся в одной плоскости, $\left\{\mathbf{e}_{i}\right\}$ имеют компоненты $\mathbf{e}_{3, x}, \mathbf{e}_{1, x z}, \mathbf{e}_{2, x y z}$. По виду тензора $\chi_{i j k}^{(2)}$ (4) и значениям параметра $v$ можно заключить, что в предложенной модели $\operatorname{Re} \chi^{(2)}>\operatorname{Im} \chi^{(2)}$. В таблице представлены полученные по известным тензорам $\chi_{i j k}^{(2)}, \varepsilon_{i j},\left\{\mathbf{k}_{i}\right\}$ и $\left\{\mathbf{e}_{i}\right\}$ значения параметров $\sigma_{1}$ и $\delta_{1}$ (3) для плазмы. Данные результаты позволяют при заданной интенсивности ВН оценить характерную длину эффективной передачи мощности от $\mathrm{BH}$ к СВ, на которой происходит усиление амплитуды волны в $e$ раз $\left(r_{S}, \tilde{r}_{S}\right.$ при $\Delta k=0$ и $\Delta k \neq 0$ соответственно). Зависимость параметров $\left\{\sigma_{n}\right\}$ от конфигурации $\left\{\mathbf{k}_{i}\right\},\left\{\mathbf{e}_{i}\right\}$ весьма консервативна, что дает возможность провести достаточно общую оценку условий реализации ПГИ для лабораторной плотной плазмы с аппроксимацией $\chi_{i j k}^{(2)}$ в форме (4).

В таблице представлены данные параметров ПГИ для полностью однократно ионизованной плазмы различной концентрации, оцененные по предложенной выше модели. Заметим, что условие $f=\infty$ (условие фазового синхронизма [9]) в плазме не может быть в точности выполнено ввиду резкой зависимости показателя преломления плазмы от частоты. Тем не менее использование (1), (2) при $\Delta k \rightarrow 0$ для качественного обсуждения условий возникновения ПГИ в лабораторной плазме является приемлемым (ср. $r_{S}, \tilde{r}_{S}$ при $f=\infty, 2$ в таблице). Анализ полученных значений параметров показывает, что квадратичное взаимодействие электромагнитных волн в пространственно однородной плазме во внешнем постоянном и однородном магнитном поле при достижимых в эксперименте значениях полей $B_{0}$ и $a_{3}(0)$ (такие поля не провоцируют „нагревную“ и „стрикционную“ неустойчивости [11]) приводит к реализации явления ПГИ на $r_{S} \sim 1 \mathrm{~cm}$ лишь для достаточно умеренных концентраций заряженных частиц. Это связано как с весьма малыми значениями функций нелинейной реакции $\left\{\sigma_{n}\right\}$, так и с высоким уровнем поглощения излучения в более плотной плазме. Другими словами, реализуется своего рода „отсечка“ ПГИ в неидеальной плазме. В случае ГВГ „отсечка“ соответствует нарушению условия $\sigma_{2} a_{1}^{2} \cos (\Delta k z)-\delta_{2} a_{2}>0$ (см. выше) в сильно или полностью ионизованной плотной плазме. Как показывает проведенный анализ, для преодоления „отсечки“ нужно увеличивать интенсивность воздействия излучения на среду, что может привести к другому типу взаимодействия излучения с веществом (см., например, [12]).

Таким образом, в работе впервые рассмотрены явления ПГИ и ГВГ в плотной лабораторной плазме в постоянном магнитном поле, вызванные квадратичным взаимодействием электромагнитных волн. Применен модельный подход, основанный на использовании явных аппроксимаций для функций реакции с подгоночными параметрами, которые найдены из точных частотных моментов этих функций. Предложенный подход показал удовлетворительное совпадение с экспериментальными данными по линейным оптическим характеристикам плотной плазмы в широком диапазоне параметров [7]. В рамках предложенной модели обнаружена „отсечка“ явлений ПГИ и ГВГ, обусловленная конкуренцией генерации полем накачки квадратичного отклика в плазме и его поглощения.

\section{Финансирование работы}

Работа выполнена по теме государственного задания № 0089-2019-0002.

\section{Конфликт интересов}

Автор заявляет, что у него нет конфликта интересов.

\section{Список литературы}

[1] Goldberg B.M., Chng T.L., Dogariu A., Miles R.B. // Appl. Phys. Lett. 2018. V. 112. P. 064102.

[2] Zhang Y., Huang D., Shan Y., Jiang T., Zhang Z., Liu K., Shi L., Cheng J., Sipe J.E., Liu W.-T., Wu S. // Phys. Rev. Lett. 2019. V. 122. P. 047401.

[3] Wang J., Jin K., Yao H., Gu J., Xu X., Ge C., Wang C., He M., Yang G. // Appl. Phys. Lett. 2018. V. 112. P. 102904. 
[4] Iwai A., Sakai O., Omura Y. // Phys. Plasmas. 2017. V. 24. P. 122112

[5] Pavlov G.A. // Europhys. Lett. 2015. V. 110. P. 45001; Pavlov G.A., Troshchiev Yu.V. // Contrib. Plasma Phys. 2015. V. 55. N 2-3. P. 254-263.

[6] Павлов Г.А. // Письма в ЖТФ. 2007. Т. 33. В. 2. С. 36-42; Pavlov G.A. // Europhys. Lett. 2008. V. 83. P. 35002.

[7] Павлов Г.А. Процессы переноса в плазме с сильным кулоновским взаимодействием. М.: Энергоатомиздат, 1995. 192 c. [Pavlov G.A. Transport processes in plasmas with strong Coulomb interaction. Amsterdam: Gordon \& Breach, 2000. 200 p.].

[8] Book of Abstracts of SCCS 2017. Kiel, Germany, 2017. 169 p. http://www.uni-kiel.de/sccs2017_book_of_abstracts.pdf

[9] Дмитриев В.Г., Тарасов Л.В. Прикладная нелинейная оптика. М.: Физматлит, 2004. 512 с.

[10] Ситенко А.Г. Флуктуации и нелинейное взаимодействие волн в плазме. Киев: Наук. думка, 1977. 248 с.; Мейснер Л.Б., Салтиел С.М. // Нелинейные восприимчивости: экспериментальные данные и методы расчета. Справочник по лазерам / Под ред. А.М. Прохорова. М.: Сов. радио, 1978. Т. 2. С. 271-292.

[11] Геккер И.Р. Взаимодействие сильных электромагнитных полей с плазмой. М.: Атомиздат, 1978. 312 с.

[12] Li B.Y., Liu F., Chen M., Chen Z.Y., Yuan X.H., Weng S.M., Jin T., Rykovanov S.G., Wang J.W., Sheng Z.M., Zhang J. // Phys. Rev. E. 2019. V. 100. P. 053207. 